Pacific Journal of Mathematics

INTEGRALS OF FOLIATIONS ON MANIFOLDS WITH A 


\title{
INTEGRALS OF FOLIATIONS ON MANIFOLDS WITH A GENERALIZED SYMPLECTIC STRUCTURE
}

\author{
R. O. Fulp and J. A. Marlin
}

Let $M$ be a $C^{\infty}$ manifold of dimension $m$ and $E$ an integrable subbundle (foliation) of the tangent bundle $T M$. We are interested in structures on the set of all local integrals of $E$. For example, if $M$ is a symplectic manifold then the Poisson brackets operation on the set $C_{10 c}^{\infty}$ of all local functions of $M$ defines an algebraic structure on $C_{1 \circ}^{\infty}$. Earlier authors have called such structures "function groups." In particular, if $X_{H}$ is a nonvanishing Hamiltonian vector field, then $X_{H}$ defines a foliation $E$ of $M$ and the set of all local integrals of $E$ is also a function group.

The Poisson brackets operation can be defined on manifolds with somewhat less restrictive requirements than that of being symplectic. Other authors such as S. Lie and C. Carathéodory [4] have studied this more general notion of Poisson brackets in the classical local setting. Hermann [9, p. 31] has indicated how to extend the definition of Poisson brackets to functions on manifolds having a closed 2-form $\omega$ of constant rank (Recall that $M$ is called symplectic if $\omega_{p}$ has rank $m$ for each $p \in M$ ).

The paper is largely self-contained, but does require the use of the following basic identities:

$$
L_{X} Y=[X, Y], \quad L_{X}=i_{X} d+d i_{X}, \quad L_{X} i_{Y}-i_{Y} L_{X}=i_{[X, Y]} .
$$

The proofs of these identities may be found in Chapter IV of the first volume of [7]. Other undefined terms appear either in [1] or [7].

1. Generalized symplectic structures on manifolds. Let $M$ be a $C^{\infty}$ manifold of dimension $m$ and let $\omega$ be a closed 2 -form on $M$. Recall that the kernel of a 2 -form $\omega$ can be defined at each point $p \in M$ by

$$
\begin{aligned}
\operatorname{ker} \omega_{p} & =\left\{v \in M_{p} \mid \omega\left(v, M_{p}\right)=0\right\} \\
& =\left\{v \in M_{p} \mid \omega\left(M_{p}, v\right)=0\right\} .
\end{aligned}
$$

The rank of $\omega$ at $p$ is defined to be the rank of the bilinear map $\omega_{p}: M_{p} \times M_{p} \rightarrow R$. Of course, since $\omega_{p}$ is a skew-symmetric bilinear map its rank is the even integer $m-\operatorname{dim}\left(\operatorname{ker} \omega_{p}\right)$.

Let $\Gamma$ denote the set of sections of $T M$ and $\Gamma^{*}$ the set of sections of $T^{*} M$. Define $\alpha: \Gamma \rightarrow \Gamma^{*}$ by 


$$
\alpha_{X}=i_{X} \omega
$$

Let $\Gamma_{\omega}=\left\{X \in \Gamma \mid i_{X} \omega=0\right\}=\operatorname{ker} \alpha$.

If we fix $p \in M$ then we may regard $\alpha=\alpha_{p}$ as a map from $T_{p} M$ into $T_{p}^{*} M$. Since $T_{p} M$ is finite dimensional, $T_{p} M \cong T_{p}^{*} M$ and we may apply the standard duality theorems of linear algebra. Thus, if we use the usual pairing between $T_{p} M$ and $T_{p}^{*} M$ we have, for $x, y \in T_{p} M$,

$$
\langle\alpha(y), x\rangle=\alpha(y)(x)=\omega_{p}(y, x)=-\omega_{p}(x, y)=\left\langle y^{* *},-\alpha(x)\right\rangle .
$$

Thus $\alpha$ is skew adjoint: $\alpha^{*}=-\alpha$, and

$$
\operatorname{im}\left(\alpha^{*}\right)=\operatorname{im}(\alpha)=\operatorname{ker}(\alpha)^{\perp}
$$

where $\operatorname{ker}(\alpha)^{\perp}$ is the annihilator of $\operatorname{ker}(\alpha)$ in $T_{p}^{*} M$.

From this we see that if $\Gamma_{\omega}^{*} \equiv\left\{\beta \in \Gamma^{*} \mid \beta\left(\Gamma_{\omega}\right)=0\right\}$, then $\Gamma_{\omega}^{*}=$ $\operatorname{ker}(\alpha)^{\perp} \leqq \Gamma^{*}$. From these remarks it follows that $\Gamma_{\omega}^{*}=\operatorname{im}(\alpha)$.

If $\operatorname{inv}(\Gamma)$ is defined by $\operatorname{inv}(\Gamma)=\left\{X \in \Gamma \mid L_{X} \Gamma_{\omega} \subseteq \Gamma_{\omega}\right\}$ then $\operatorname{inv}(\Gamma)$ is the normalizer of $\Gamma_{\omega}$ in $\Gamma$ and thus is a Lie subalgebra of $\Gamma$. Moreover, it is immediate from the definitions any subalgebra of a Lie algebra is always an ideal in its normalizer, thus $\Gamma_{\omega}$ is an ideal in inv $(\Gamma)$. We summarize all these remarks as a proposition.

Proposition 1.1. The image of the map $\alpha: \Gamma \rightarrow \Gamma^{*}$ is precisely

$$
\Gamma_{\omega}^{*} \equiv\left\{\beta \in \Gamma^{*} \mid \beta\left(\Gamma_{\omega}\right)=0\right\} \text {. }
$$

Moreover, $\operatorname{inv}(\Gamma) \equiv\left\{X \in \Gamma \mid L_{X} \Gamma_{\omega} \subseteq \Gamma_{\omega}\right\}$ is a Lie subalgebra of $\Gamma$ which contains $\Gamma_{\omega}$ as an ideal.

We now want to show that $\alpha \mid \operatorname{inv}(\Gamma)$ is a Lie algebra antihomomorphism from $\operatorname{inv}(\Gamma)$ onto the $\operatorname{set} \operatorname{inv}\left(\Gamma_{\omega}^{*}\right) \leqq \Gamma^{*}$ where $\operatorname{inv}\left(\Gamma_{\omega}^{*}\right)$ is defined by

$$
\operatorname{inv}\left(\Gamma_{\omega}^{*}\right) \equiv\left\{\beta \in \Gamma_{\omega}^{*} \mid L_{Z} \beta=0 \text { for all } Z \in \Gamma_{\omega}\right\} .
$$

Before doing this we need to define a Lie algebra structure on $\operatorname{inv}\left(\Gamma_{\omega}^{*}\right)$. For this we need a lemma.

LEMMA 1.2. If $Z \in \Gamma_{\omega}$, then $L_{Z} \Gamma_{\omega}^{*} \subseteq \Gamma_{\omega}^{*}$. In fact, $L_{Z} \alpha_{X}=\alpha_{L_{Z} X}$ for each $X \in \Gamma$.

Proof. Since $L_{Z} \omega=\left(i_{z} d+d i_{z}\right) \omega=0, L_{Z} \alpha_{X}=L_{Z} i_{X} \omega=i_{X} L_{Z} \omega+$ $i_{[X, Z]} \omega=\alpha_{[Z, X]}=\alpha_{L_{Z} X}$

Corollary 1.3. $\alpha(\operatorname{inv} \Gamma)=\operatorname{inv}\left(\Gamma_{\omega}^{*}\right)$.

Proof. From Proposition 1.1, we know that inv $\left(\Gamma_{\omega}^{*}\right)$ is contained 
in $\operatorname{im}(\alpha)$. By the lemma above, for $Z \in \Gamma_{\omega}, L_{Z} \alpha_{X}=\alpha_{L_{Z} X}=-\alpha_{L_{X} Z}$; thus $\alpha_{X} \in \operatorname{inv}\left(\Gamma_{\omega}^{*}\right)$ iff $L_{X} Z \in \Gamma_{\omega}$ for all $Z \in \Gamma_{\omega}$. It follows that $\alpha(\operatorname{inv} \Gamma)=\operatorname{inv}\left(\Gamma_{\omega}^{*}\right)$.

The map $\alpha$ is a linear transformation from inv $(\Gamma)$ onto inv $\left(\Gamma_{\omega}^{*}\right)$ with kernel $\Gamma_{\omega}$. Thus $\operatorname{inv}\left(\Gamma_{\omega}^{*}\right) \cong \operatorname{inv}(\Gamma) / \Gamma_{\omega}$ as vector spaces. Since $\Gamma_{\omega}$ is a Lie ideal in inv $(\Gamma)$, the quotient inv $(\Gamma) / \Gamma_{\omega}$ is a Lie algebra. We impose this Lie structure on $\operatorname{inv}\left(\Gamma_{\omega}^{*}\right)$ via the vector space isomorphism induced by $\alpha$.

Proposition 1.4. The set inv $\left(\Gamma_{\omega}^{*}\right)$ of all invariant elements of $\Gamma_{\omega}^{*}$ is a Lie algebra under $\{$,$\} where \{$,$\} is defined by$

$$
\left\{\alpha_{X}, \alpha_{Y}\right\}=-\alpha_{[X, Y]} .
$$

The $\operatorname{map} \alpha: \operatorname{inv}(\Gamma) \rightarrow \operatorname{inv}\left(\Gamma_{\omega}^{*}\right)$ is a Lie algebra antihomomorphism with kernel $\Gamma_{\omega}$, thus the sequence

$$
0 \longrightarrow \Gamma_{\omega} \longrightarrow \operatorname{inv}(\Gamma) \stackrel{\alpha}{\longrightarrow} \operatorname{inv}\left(\Gamma_{\omega}^{*}\right) \longrightarrow 0,
$$

is an exact sequence of Lie algebras.

REMARK. It is easy to see that for $\alpha, \beta \in \operatorname{inv}\left(\Gamma_{\omega}^{*}\right)$ one has

$$
\left.\{\alpha, \beta\}\right|_{U}=\left\{\left.\alpha\right|_{U},\left.\beta\right|_{U}\right\}
$$

for open subsets $U$ of $M$.

REMARK. We now call attention to certain identities which have proven useful in our work. If $\beta$ and $\gamma$ are closed 1-forms in $\Gamma_{\omega}^{*}$ and $X$ and $Y$ are vector fields such that $\beta=\alpha_{X}, \gamma=\alpha_{Y}$, then

$$
\{\beta, \gamma\}=-i_{[X, Y]} \omega=-L_{X} Y=L_{Y} \beta=d(2 \omega(X, Y)) .
$$

Note, in particular, that $\{\beta, \gamma\}$ is exact.

To see that the above identities hold, observe that

$$
\begin{aligned}
\{\beta, \gamma\} & =\left\{\alpha_{X}, \alpha_{Y}\right\}=-\alpha_{[X, Y]} \\
& =-i_{[X, Y]} \omega=-L_{X} i_{Y} \omega+i_{Y} L_{X} \omega \\
& =-L_{X} \alpha_{Y}+i_{Y}\left(d i_{X}+i_{X} d\right) \omega=-L_{X} \alpha_{Y}+i_{Y}\left(d \alpha_{X}\right) \\
& =-L_{X} \gamma=-\left(d i_{X}+i_{X} d\right) \gamma=-d\left(i_{X} \gamma\right) \\
& =2 d(\omega(X, Y)) .
\end{aligned}
$$

Let $C^{\infty}(\omega)$ denote the set of all invariant functions of $\operatorname{ker} \omega$, i.e.

$$
C^{\infty}(\omega)=\left\{f \mid L_{Z} f=d f(Z)=0 \text { for all } Z \in \Gamma_{\omega}\right\} .
$$

We now define the Poisson bracket $\{$,$\} for pairs of invariant$ functions of $\operatorname{ker} \omega$ : 


$$
\{f, g\}=2 \omega\left(X_{f}, X_{g}\right)
$$

where $X_{f}$ and $X_{g}$ are any two vector fields such that

$$
d h=i_{X_{h}} \omega
$$

for $h=f, g$. Clearly $\{$,$\} is well-defined.$

Proposition 1.5. If $f, g \in C^{\infty}(\omega)$ the following statements are true:

(1) $\{f, g\}=-L_{X_{f}}(g)=L_{X_{g}}(f)$

(2) $d\{f, g\}=\{d f, d g\}$.

Moreover, $C^{\infty}(\omega)$ is a Lie algebra with respect to $\{$,$\} and$

(3) $X_{\{f, g\}}+\left[X_{f}, X_{g}\right] \in \Gamma_{\omega}$.

Proof. If $f, g \in C^{\infty}(\omega)$ then (1) follows from $\{f, g\}=2 \omega\left(X_{f}, X_{g}\right)=$ $d f\left(X_{g}\right)=L_{X_{g}}(f)$. By the above remark we have $d\{f, g\}=d\left(2 \omega\left(X_{f}\right.\right.$, $\left.\left.X_{g}\right)\right)=\{d f, d g\}$ and thus (2) follows. The statement (3) is immediate from definitions.

Proposition 1.6. If $f, g \in C^{\infty}(\omega)$ and $d g=i_{X_{g}} \omega$ then $f$ is constant on integral curves of $X_{g}$ iff $\{f, g\}=0$.

Proof. $X_{g}(f)=L_{X_{g}}(f)=\{f, g\}=0$.

2. Function groups. Let $M$ be a connected $C^{\infty}$-manifold of dimension $m$ with a 2 -form $\omega$ of constant rank $\rho \leqq m$. In this case $\operatorname{ker} \omega$ is locally trivial, i.e., $\operatorname{ker} \omega$ is a subbundle of TM. Moreover, ker $\omega$ is actually an integrable subbundle of $T M$ and thus is a foliation of $M$. To see this observe that for $X \in \Gamma_{\omega}$,

$$
L_{X} \omega=i_{X}(d \omega)+d\left(i_{X} \omega\right)=0 .
$$

Thus for $X, Y$ in $\Gamma_{\omega}$,

$$
i_{[X, Y]} \omega=L_{X}\left(i_{Y} \omega\right)-i_{Y}\left(L_{X} \omega\right)=0 .
$$

A function $f$ is called a local $C^{\infty}$ function on $M$ iff the domain $U=\operatorname{dom}(f)$ of $f$ is an open subset of $M$ and $f \in C^{\infty}(U)$. Let $C_{\text {loc }}^{\infty}=C_{\text {loc }}^{\infty}(M)$ denote the set of all local $C^{\infty}$ functions of $M$. Let $C_{\mathrm{loc}}^{\infty}(\omega)$ denote the set of all local integrals of the foliation $\operatorname{ker} \omega$, i.e.,

$$
C_{\mathrm{loc}}^{\infty}(\omega)=\left\{f \in C_{\mathrm{loc}}^{\infty} \mid d f\left(\operatorname{ker}\left(\omega_{p}\right)\right)=0 \text { for all } p \in \operatorname{dom} f\right\} .
$$

Note that in the symplectic case $C_{1 \mathrm{loc}}^{\infty}(\omega)=C_{\mathrm{loc}}^{\infty}$.

Recall that a function $f \in C_{\mathrm{loc}}^{\infty}$ is said to be $C^{\infty}$-dependent on $f_{1}, f_{2}, \cdots, f_{r} \in C_{\text {loc }}^{\infty}$ at $p \in M$ provided that there is a neighborhood $U$ 
of $p$ and a function $F \in C_{\text {loc }}^{\infty}\left(R^{r}\right)$ such that

(1) the functions $f, f_{1}, f_{2}, \cdots, f_{r}$ are all defined on $U$, and

(2) $f(x)=F\left(f_{1}(x), f_{2}(x), \cdots, f_{r}(x)\right)$ for each $x \in U$.

If $f, g \in C_{\mathrm{loc}}^{\infty}(\omega)$ and $U=\operatorname{dom} f \cap \operatorname{dom} g \neq \varnothing$, then $U$ can be regarded as a manifold with $\left.\omega\right|_{U}$ a 2 -form of constant rank on $U$. Thus $\{f, g\}=\{f|U, g| U\}$ is a well-defined element of $C^{\infty}(\omega \mid U)$. It follows that $X_{f}$ and $X_{g}$ have domains dom $f$ and dom $g$ respectively and thus $\left[X_{f}, X_{g}\right]$ and $X_{\{f, g\}}$ are well-defined vector fields on $U$. Similarly, $\{d f, d g\}$ is a well-defined 1-form on $U$.

Definition 2.1. A nonvoid subset $\mathscr{S}$ of $C_{\mathrm{loc}}^{\infty}(\omega)$ is called a function group iff the following conditions hold:

(1) $M=\bigcup_{f \in \mathscr{S}} \operatorname{dom}(f)$,

(2) if $f \in \mathscr{S}$ and $U$ is an open subset of $\operatorname{dom} f$ then $f \mid U \in \mathscr{S}$,

(3) if $f, g \in \mathscr{S}$ and $\operatorname{dom}(f) \cap \operatorname{dom}(g) \neq \phi$, then $\{f, g\} \in \mathscr{S}$,

(4!) if $f_{1}, f_{2}, \cdots, f_{k}$ are elements of $\mathscr{S}$ and $f$ is $C^{\infty}$-dependent on $f_{1}, f_{2}, \cdots, f_{k}$ then $f \in \mathscr{S}$,

(5) Let $U=\bigcup_{j} U_{j}$ where $U_{j}$ is an open subset of $M$ for each $j$. If $f \in C^{\infty}(U)$ and $f \mid U_{j} \in \mathscr{S}$, for each $j$, then $f \in \mathscr{S}$.

A function group is said to be of rank $r$ at a point $p \in M$ provided that there are $r$ functions $f_{1}, f_{2}, \cdots, f_{r}$ in $\mathscr{S}$ such that

(1) there is a neighborhood $U$ of $p$ contained in the domain of each of the functions $f_{1}, f_{2}, \cdots, f_{r}$ such that for each $q \in U$

$$
d f_{1 q}, d f_{2 q}, \cdots, d f_{r q}
$$

are independent elements of $M_{q}^{*}$, and

(2) for each $f \in \mathscr{S}$, with $p \in \operatorname{dom} f, f$ is $C^{\infty}$-dependent on $f_{1}, f_{2}$, $\cdots, f_{r}$ on some neighborhood of $p$.

In case $f_{1}, f_{2}, \cdots, f_{r}$ satisfy (1) and (2) we say that $f_{1}, f_{2}, \cdots, f_{r}$ generate $\mathscr{S}$ at $p$.

REMARK. If $f_{1}, f_{2}, \cdots, f_{r}$ generate $\mathscr{S}$ at $p$ and $g_{1}, g_{2}, \cdots, g_{s}$ generate $\mathscr{S}$ at $p$, then $r=s$. To see this observe that the definition implies that there exists functions $F_{i} \in C_{\text {loc }}^{\infty}\left(\boldsymbol{R}^{r}\right), G_{j} \in C_{\mathrm{loc}}^{\infty}\left(\boldsymbol{R}^{r}\right)$ such that for $i=1,2, \cdots, s$ and $j=1,2, \cdots, r$

$$
g_{i}=F_{i}\left(f_{1}, \cdots, f_{r}\right) \text { and } f_{j}=G_{j}\left(g_{1}, \cdots, g_{s}\right) .
$$

Then the chain rule applied to the equalities

$$
\begin{aligned}
& g_{i}=F_{i}\left(G_{1}\left(g_{1}, \cdots, g_{s}\right), \cdots, G_{r}\left(g_{1}, \cdots, g_{s}\right)\right) \\
& f_{j}=G_{j}\left(F_{1}\left(f_{1}, \cdots, f_{r}\right), \cdots, F_{s}\left(f_{1}, \cdots, f_{r}\right)\right)
\end{aligned}
$$

implies that $\left(\partial F_{i} / \partial f_{j}\right)$ and $\left(\partial G_{k} / \partial g_{l}\right)$ are inverse matrices. Hence $r=. s$ 
REMARK. If $\mathscr{S}$ is a function group of rank $r$ at $p \in M$, then one can easily show that if $h_{1}, h_{2}, \cdots, h_{r}$ are elements of $\mathscr{S}$ such that $d h_{1 p}, d h_{2 p}, \cdots, d h_{r p}$ are independent in $M_{p}^{*}$ then they generate $\mathscr{S}$ at $p$.

A function group is said to be of rank $r$ iff it is of rank $r$ at each point of $M$.

The following is an example to show that a function group may not have the same rank at each point of $M$. Let $M=R^{2}$ and $\omega=d x \wedge d y$. Let $f \in C^{\infty}(R)$ such that

$$
f(x)=0, x \leqq 0 \text { and } f(x)>0, x>0 .
$$

Define functions $F$ and $G$ on $R^{2}$ by $F(x, y)=x$ and $G(x, y)=f(x) y$. Let $\mathscr{S}$ denote the set of all functions of the form

$$
(x, y) \longrightarrow \Phi(F(x, y), G(x, y))
$$

where $\Phi$ is any element of $C_{\mathrm{loc}}^{\infty}\left(\boldsymbol{R}^{2}\right)$. Then $\mathscr{S}$ is a function group which has rank 2 at points $(x, y)$ where $x>0$ and rank 1 at points $(x, y)$ where $x<0$.

We describe the relation between function groups of rank $r$ and foliations.

THEOREM 2.2. Let $\mathscr{S}$ be a function group of rank $r$ and let $E_{p}=\left\{X_{p} \mid 2 \omega_{p}\left(X_{p}, \cdot\right)=d f(\cdot)\right.$ for $\left.f \in \mathscr{S}\right\}$ for each $p \in M$. Then $E=$ $\mathrm{U}_{p \in M} E_{p} \leqq T M$ is an integrable subbundle of $T M$ which contains $\operatorname{ker}(\omega)$.

Proof. We show $E$ is locally trivial. Choose $p \in M, U$ a neighborhood of $p$, and $f_{1}, \cdots, f_{r}$ in $\mathscr{S}$ as in the definition of a generating set for $\mathscr{S}$ at $p$. Let $X_{i}=X_{f_{i}}$. If $q \in U$ and $v \in E_{q}$ then $v=\left(X_{h}\right)_{q}$ for some $h \in \mathscr{S}$. Since $d f_{1 q}, \cdots, d f_{r q}$ are independent we know that there exists $F \in C_{10 \mathrm{c}}^{\infty}\left(\boldsymbol{R}^{r}\right)$ such that

$$
h=F\left(f_{1}, \cdots, f_{r}\right)
$$

on a neighborhood $V$ of $q$. One sees that

$$
X_{h}-\sum_{1}^{r} \frac{\partial F}{\partial x_{i}} X_{i} \in \Gamma(\operatorname{ker}(\omega \mid V))
$$

and thus $v=\left(X_{h}\right)_{q} \in\left\langle X_{1 q}, \cdots, X_{r q}\right\rangle+\operatorname{ker}\left(\omega_{q}\right)$. Therefore $E$ is a subbundle of $T M$.

We show $E$ is integrable. Let $X, Y$ belong to $\Gamma(E)$ and let $p \in M$. On a neighborhood $U$ of $p$ both $X$ and $Y$ are of the form

$$
\Sigma \lambda_{i} X_{i}+Z
$$


for $\lambda_{i} \in C^{\infty}(U), Z \in \Gamma\left(\left.\omega\right|_{U}\right)$, and $X_{i}=X_{f_{i}}$. Then $[X, Y]$ will be in $\Gamma(E)$ provided that for $1 \leqq i, j \leqq r,\left[X_{i}, X_{j}\right] \in \Gamma(E)$ and for $Z \in \Gamma_{(\omega \mid U)}$, $\left[X_{i}, Z\right] \in \Gamma(E)$. Since $\mathscr{S}$ is a function group, $\left\{f_{i}, f_{j}\right\} \in \mathscr{S}$ and $X_{\left\{f_{i}, f_{j}\right\}} \in \Gamma(E \mid U)$. By (3) of Proposition 1.5 it follows that $\left[X_{i}, X_{j}\right] \epsilon$ $\Gamma(E \mid U)$. Moreover, $2 \omega\left(\left[Z, X_{j}\right], Y\right)=\left(i_{\left[Z, X_{j}\right]} \omega\right)(Y)=L_{Z}\left(i_{X_{j}} \omega\right)(Y)=$ $L_{Z}\left(d f_{j}\right)(Y)=d\left(i_{z} d f_{j}\right)(Y)=0$ for all $Y \in \Gamma$. Thus $\left[Z, X_{j}\right] \in \Gamma_{\omega}$ for each $Z \in \Gamma_{\omega}$ and consequently $E$ is integrable.

Hereafter the foliation $E$ described above will be called the foliation determined by $\mathscr{S}$.

If $\mathscr{S}$ is a function group then the reciprocal of $\mathscr{S}$ is defined to be the set of all $\mathrm{g} \in C_{\mathrm{loc}}^{\infty}(\omega)$ such that $\{f, g\}=0$ for all $f \in \mathscr{S}$ such that $\operatorname{dom} f \cap \operatorname{dom} g \neq \phi$. We denote the reciprocal of $\mathscr{S}$ by $\mathscr{S}^{\prime}$. The fact that $\mathscr{S}^{\prime}$ is a function group is somewhat trivial. To see that $\mathscr{S}^{\prime}$ is closed under $\{$,$\} one uses the Jacobi identity.$ To see that (4) of Definition 2.1 holds we need an identity which is useful in subsequent sections of our paper: for arbitrary $h_{1}, h_{2}, \cdots$, $h_{n} \in C_{\mathrm{loc}}^{\infty}(\omega)$ and $F \in C_{\mathrm{loc}}^{\infty}\left(\boldsymbol{R}^{n}\right)$, then

$$
\left\{f, F\left(h_{1}, h_{2}, \cdots, h_{n}\right)\right\}=\sum_{i} \frac{\partial F}{\partial h_{\imath}}\left(h_{1}, h_{2}, \cdots, h_{n}\right)\left\{f, h_{i}\right\} .
$$

Part (4) follows immediately from this identity. To prove 2.4 observe that

$$
\begin{aligned}
\left\{f, F\left(h_{1}, h_{2}, \cdots, h_{n}\right)\right\} & =-2 \omega\left(X_{F}, X_{f}\right)=-d F\left(X_{f}\right) \\
& =-\sum_{i} \frac{\partial F}{\partial h_{i}}\left\{h_{i}, f\right\}=\sum_{i} \frac{\partial F}{\partial h_{\imath}}\left\{f, h_{i}\right\} .
\end{aligned}
$$

REMARK. It is obvious that $\mathscr{S} \subseteq \mathscr{S}^{\prime \prime}$ for any function group $\mathscr{S}$. Observe that if $\mathscr{S}$ has rank $r$, then $\mathscr{S}=\mathscr{S}^{\prime \prime}$.

If $\mathscr{S}$ is a function group then $\mathscr{T}$ is a subgroup of $\mathscr{S}$ iff $\mathscr{T}$ is a function group such that $\mathscr{T} \cong \mathscr{S}$.

Observe that every function group is a subgroup of the function group $C_{\mathrm{loc}}^{\infty}(\omega)$. Also the intersection of two subgroups is a subgroup. In particular $\mathscr{S} \cap \mathscr{S}^{\prime}$ is a subgroup of both $\mathscr{S}$ and $\mathscr{S}^{\prime}$.

Proposition 2.6. Let $\mathscr{S}$ be a function group of rank $r$ at $p$. Then its reciprocal has rank $\rho-r$ at $p$.

Proof. Let $p \in M$ and let $f_{1}, \cdots, f_{r}$ be generators of $\mathscr{S}$ at $p$. Choose coordinates $x_{1}, \cdots, x_{m}$ at $p$ such that $X_{i}=X_{f_{i}}=\partial / \partial x_{i}$ for $1 \leqq i \leqq r$ and such that $\left\{\partial / \partial x_{r+j}\right\} 1 \leqq j \leqq m-p$ generate $\Gamma_{\omega}$ near $p$. Then any integral of the integrable system $X_{1}, \cdots, X_{r}, \partial / \partial x_{r+1}$, $\cdots, \partial / \partial x_{r+m-\rho}$ depends only on the last coordinates. Since each 
$f \in \mathscr{S}^{\prime}$ is an integral of this system it follows that $x_{m+r-\rho+1}, \cdots x_{m}$ generates $\mathscr{S}^{\prime}$ at $p$.

Using arguments similar to those above we obtain the following corollary.

COROLlaRY 2.7. Let $\mathscr{S}$ be a function group of rank $r, \mathscr{S}^{\prime}$ the reciprocal of $\mathscr{S}$, and $E$ the foliation determined by $\mathscr{S}$. Then

(1) $E_{p}=\cap\left\{\operatorname{ker} d g_{p} \mid g \in \mathscr{S}^{\prime}\right\}$, for each $p \in M$,

(2) if $g_{1}, g_{2}, \cdots, g_{\rho-r}$ generate $\mathscr{S}^{\prime}$ at $p \in M$, then there is a neighborhood $U$ of $p$ such that the map $x \rightarrow\left(g_{1}(x), g_{2}(x), \cdots, g_{\rho_{-r}}(x)\right)$ $i s$ constant on each leaf of the foliation $E \mid U$ of $U$.

We say that a subbundle $E$ of $T M$ is locally Hamiltonian iff $\operatorname{ker}(\omega) \subseteq E$ and for each $p \in M$ there is a neighborhood $U$ of $p$ such that $\Gamma(E \mid U)$ is spanned by vector fields $X$ which satisfy $d f=i_{X} \omega$ for some $f \in C_{\mathrm{loc}}^{\infty}(\omega)$.

Proposition 2.8. An integrable subbundle $E$ is the foliation determined by some function group iff $E$ is locally Hamiltonian. Moreover, the function group which determines such an $E$ is unique.

Proof. Clearly if $E$ is determined by some function group, then $E$ is locally Hamiltonian.

Conversely, suppose that $E$ is locally Hamiltonian and consider the set $\mathscr{F}$ of all local integrals of $E$. We now show that $\mathscr{F}$ is a function group and that $E$ is determined by the reciprocal, $\mathscr{S}^{\prime}$, of $\mathscr{F}$. Let $f, g \in \mathscr{F}, p \in M$, and $X \in \Gamma(E)$. There is no loss of generality in assuming that there is an $H \in C_{\mathrm{loc}}^{\infty}(\omega)$ such that $2 \omega(X, \cdot)=d H(\cdot)$ in a neighborhood of $p$. It follows that

$$
\begin{aligned}
d\{f, g\}(X) & =L_{X_{H}}(\{f, g\})=\{f,\{g, H\}\}+\{g,\{H, f\}\} \\
& =\left\{L_{X} g, f\right\}+\left\{L_{X} f, g\right\}=0
\end{aligned}
$$

by Proposition 1.5, the Jacobi identity, and the fact that $X \in \Gamma(E)$. Thus $\{f, g\} \in \mathscr{J}$ and it follows that $\mathscr{J}$ is a function group with constant rank. Since $\mathscr{I}=\mathscr{I}^{\prime \prime}$ it follows from Corollary 2.7 that

$$
E=\cap\left\{\operatorname{ker} d f \mid f \in \mathscr{J}^{\prime \prime}=\mathscr{I}\right\} .
$$

REMARK. If $\mathscr{S}$ is any function group then $\mathscr{S}$ determines a unique integrable locally Hamiltonian subbundle $E$ of $T M$ and conversely. If $E$ is determined by $\mathscr{S}$ then the reciprocal of $\mathscr{S}$ is precisely the set of all local integrals of $E$. If $E$ is an integrable locally Hamiltonian subbundle of $T M$ then the set of all local inte- 
grals of $E$ is a function group. The foliation determined by the reciprocal of this function group is precisely $E$.

Let $\mathscr{S}$ be a function group of rank $r$. We say that a set $S \subseteq C^{\infty}(M)$ globally generates $\mathscr{S}$ provided that for each $p \in M$ there exist functions $f_{1}, f_{2}, \cdots, f_{r} \in S$ and a neighborhood $U$ of $p$ such that $\left\{f_{1}\left|U, f_{2}\right| U, \cdots, f_{r} \mid U\right\}$ generates $\mathscr{S}$ at $p$. We say that a set $T \cong \Gamma^{*}$ of closed 1 -forms globally generates $\mathscr{S}$ provided that for each $p \in M$ there exist forms $\beta_{1}, \cdots, \beta_{r} \in T$, a neighborhood $U$ of $p$, functions $f_{1}, f_{2}, \cdots, f_{r}$ satisfying $d f_{i}=\beta_{i}$ on $U$ for $i=1,2, \cdots, r$ such that $\left\{f_{1}\left|U, f_{2}\right| U, \cdots, f_{r} \mid U\right\}$ generates $\mathscr{S}$ at $p$.

Proposition 2.9. Suppose that there exist closed 1-forms $\beta_{1}, \beta_{2}$, $\cdots, \beta_{n}$ in $\Gamma_{\omega}^{*}$ and $r>0$ such that

(i) $\beta_{1}(p), \beta_{2}(p), \cdots, \beta_{n}(p)$ span an r-dimensional subspace of $M_{p}^{*}$ for each $p \in M$,

(ii) there exist functions $a_{i j_{k}} \in C^{\infty}(M)$ such that

$$
\left\{\beta_{i}, \beta_{j}\right\}=\sum_{k=1}^{n} a_{i j k} \beta_{k} .
$$

Then there exists a unique function group $\mathscr{S}$ of rank $r$ which is globally generated by $\left\{\beta_{1}, \beta_{2}, \cdots, \beta_{n}\right\}$. Conversely, if $\mathscr{S}$ is a function group of rank $r$ which is globally generated by $\beta_{1}, \beta_{2}, \cdots, \beta_{n}$ then conditions (i) and (ii) are satisfied.

Proof. The details of this proof are much like those of Theorem 2.2 and are left to the reader.

Recall that inv $\left(\Gamma_{\omega}^{*}\right)$ is a Lie algebra under $\{$,$\} . Observe that$ if $\alpha_{1}, \alpha_{2}, \cdots, \alpha_{n}$ are elements of $\operatorname{inv}\left(\Gamma_{\omega}^{*}\right)$ they span a finite dimensional subalgebra of $\operatorname{inv}\left(\Gamma_{\omega}^{*}\right)$ iff

$$
\left\{\alpha_{i}, \alpha_{j}\right\}=\sum_{k} c_{i j k} \alpha_{k}
$$

for constants $c_{i j_{k}} \in \boldsymbol{R}$.

We now give an application of function groups which is a slight generalization of certain well-known theorems.

THEOREMS 2.10. Let $M$ be a symplectic manifold $(\rho=m=2 N)$ and $\mathscr{S}$ a function group of rank $r$ on $M$. Suppose that the closed 1-forms $\left\{\alpha_{1}, \alpha_{2}, \cdots, \alpha_{n}\right\}$ globally generate $\mathscr{S}$ and that they span an $n$-dimensional subalgebra $\mathscr{L}$ of $\operatorname{inv}\left(\Gamma_{\omega}^{*}\right)=\Gamma\left(T^{*} M\right)$. If the vector field $X_{\alpha_{i}}$ is complete for each $i=1,2, \cdots, n$, then each leaf of the foliation determined by $\mathscr{S}$ is diffeomorphic to a homogeneous space 
$G / H$ where $G$ is the unique simply connected Lie group with Lie algebra $\mathscr{L}$ and $H$ is a closed subalgebra of $G$.

Proof. This is a consequence of a well-known theorem due to Palais [11] (see also Loos [10]). The details of the proof of Theorem 2.10 are similar to those of Theorem 1 of [2].

REMARK. Note that if we take $r=2 N-1$ we obtain a part of Theorem 1 of Andrié and Simms [2]. Note that if we take $r=N$ and assume that $\mathscr{L}$ is commutative we obtain a part of a theorem of Arnold [1] in which the leaves of the foliation turn out to be cylinders or tori (see, for example, Abraham [1, page 113]).

3. Invariant metrics and transverse structures. Let $M$ be a connected $C^{\infty}$-manifold of dimension $m$ and let $E$ be an integrable subbundle of $T M$ of dimension $r$. The normal bundle $T M / E$ of $E$ will be denoted by $Q$ and its dual $Q^{*}$ will be identified with the bundle $E^{0}$ where, for each $x \in M, E_{x}^{0}$ is the annihilator of $E_{x}$ in $T_{x}^{*} M$, i.e.,

$$
E_{x}^{0}=\left\{\beta \in T_{x}^{*} M \mid \beta\left(E_{x}\right)=0\right\} .
$$

Define a connection $\nabla^{*}$ on $\Gamma\left(E^{0}\right)$ along the leaves of $E$ by $\nabla_{X}^{*} \beta=$ $L_{X} \beta$ for $\beta \in \Gamma\left(E^{0}\right)$ and $X \in \Gamma(E)$.

Observe that if $f$ is any local integral of $E$ then $\nabla_{X}^{*}(d f)=$ $L_{X}(d f)=d f(X)=0$ and thus $d f$ is covariant constant along leaves of $E$. Also, if $f_{1}, f_{2}, \cdots, f_{m-r}$ are independent local integrals of $E$ defined on an open set $U \subseteq M$, then $d f_{1}, d f_{2}, \cdots, d f_{m-r}$ span $E^{0}$ on $U$.

LEMmA 3.1. If $\beta \in \Gamma\left(E^{0}\right)$ is closed, then $\beta$ is parallel along the leaves of $E$, i.e., $\nabla_{X}^{*} \beta=0$ for all $X \in \Gamma(E)$. $\beta \in \Gamma\left(E^{0}\right)$.

Proof. $\nabla_{X}^{*} \beta=L_{X} \beta=\left(i_{X} d\right) \beta+\left(d i_{X}\right) \beta=0$ for all $X \in \Gamma(E)$ and

CoRollary 3.2. If $\beta_{1}, \beta_{2}, \cdots, \beta_{m-r}$ are global, independent, closed elements of $\Gamma\left(E^{0}\right)$, then $E^{0}$ is parallelizable, i.e., it has $m$ $r$ global, independent, parallel sections.

If $\sigma$ is a Riemannian metric on $M$, then $Q$ may be identified with the orthogonal complement of $E$ in $T M$. Let $\sigma_{Q}=\sigma \mid(Q \times Q)$ be the induced metric on $Q$. If $\beta \in \Gamma\left(E^{0}\right)$, then $\operatorname{grad} \beta$ is that unique vector field in $\Gamma(Q)$ such that

$$
\sigma(\operatorname{grad} \beta, \cdot)=\beta
$$


and, for $\xi \in \Gamma(Q), \beta_{\xi}$ is that element of $\Gamma\left(E^{0}\right)$ defined by

$$
\beta_{\xi}=\sigma(\xi, \cdot)
$$

We define the dual connection $\nabla$ of $\nabla^{*}$ to be that connection on $\Gamma(Q)$ along leaves of $E$ such that

$$
\nabla_{X}(\xi)=\operatorname{grad}\left(\nabla_{X}^{*} \beta_{\xi}\right)
$$

for $X \in \Gamma(E)$ and $\xi \in \Gamma(Q)$. Another connection $\tilde{\nabla}$ for $\Gamma(Q)$ along the leaves of $E$ is defined by

$$
\tilde{\nabla}_{X}(\xi)=\left[L_{X} \xi\right]_{Q}
$$

where $X \in \Gamma(E), \xi \in \Gamma(\xi)$ and where $[Y]_{Q}$ denotes the component of $Y$ in $Q$.

Lemma 3.3. If $\sigma_{Q}$ is invariant with respect to $\tilde{\nabla}$ then $\tilde{\nabla}=\nabla$.

Proof. For $\xi, \eta \in \Gamma(Q)$ we have: $\left(\nabla_{X}^{*} \beta_{\xi}\right)(\eta)=\left(L_{X} \beta_{\xi}\right)(\eta)=i_{\eta}\left(L_{X} \beta_{\xi}\right)=$ $L_{X}\left(i_{\eta} \beta_{\xi}\right)-i_{[X, \eta]}\left(\beta_{\xi}\right)=L_{X}\left(\sigma_{Q}(\xi, \eta)\right)-\sigma(\xi,[X, \eta])=\left[\sigma_{Q}\left(\tilde{V}_{X} \xi, \eta\right)+\sigma_{Q}\left(\xi, \tilde{\nabla}_{X} \eta\right)\right]-$ $\sigma\left(\xi,[X, \eta]_{Q}\right)=\sigma_{Q}\left(\tilde{\nabla}_{X} \xi, \eta\right)$. Thus $\tilde{\nabla}_{X} \xi=\operatorname{grad}\left(\nabla_{X}^{*} \beta_{\xi}\right)=\nabla_{X} \xi$.

We say that $\sigma$ is invariant when $\sigma_{Q}$ is invariant with respect to the connection $\tilde{\nabla}$ in which case $\nabla=\tilde{\nabla}$. Observe that a metric $\sigma$ satisfies this property iff it is "bundle-like" in the sense of Reinhart [12]. Also the connection $\tilde{\nabla}_{X}$ can be defined for all $X \in \Gamma(T M)$ in such a way that $\tilde{\nabla}$ is a "basic connection" (see Conlon [5]). Moreover the last result is a reflection of the fact that restrictions of basic connections to $\Gamma(E)$ are unique.

LEMMA 3.4. If $\sigma$ is an invariant metric, then $\beta$ is parallel with respect to $\nabla^{*}$ iff $\operatorname{grad} \beta$ is parallel with respect to $\tilde{\nabla}$.

Proof. It is a standard result that $\beta$ is $\nabla^{*}$-parallel iff $\operatorname{grad} \beta$ is parallel relative to the dual connection $\nabla$ (see [7], Vol. II, page 342 ). Since $\nabla=\tilde{\nabla}$ the result follows.

REMARK. If $\sigma$ is an invariant metric the usual one-to-one correspondence between $\Gamma(Q)$ and $\Gamma\left(E^{0}\right)$ induces a one-to-one correspondence between $\tilde{V}$-parallel sections of $Q$ and $\nabla^{*}$-parallel sections to $E^{0}$.

REMARK. If $\xi$ and $\eta$ are $\tilde{\nabla}$-parallel along leaves of $E$ then the invariance of $\sigma$ implies that $\sigma(\xi, \eta)$ is an integral of $E$. Thus if $\beta$ is a closed element of $\Gamma^{*}(E)$ we conclude that $\sigma(\operatorname{grad} \beta, \operatorname{grad} \beta)$ is constant on leaves of $E$. If $\sigma$ is complete as well as invariant then the vector field 


$$
\frac{1}{\sigma(\operatorname{grad} \beta, \operatorname{grad} \beta)} \cdot \operatorname{grad} \beta
$$

is a complete vector field for nonvanishing closed $\beta$ in $\Gamma\left(E^{0}\right)$.

The foliation $E$ is transversally parallelizable iff there exist $m$ $r$ independent elements of $\Gamma Q$ each of which is $\tilde{\nabla}$-parallel along the leaves of $E$.

THEOREM 3.5. Suppose there exist $m-r$ everywhere independent closed 1-forms $\beta_{1}, \beta_{2}, \cdots, \beta_{m-r}$ such that

$$
\beta_{i}(\Gamma(E))=0 \text { for } \quad i=1,2, \cdots, m-r .
$$

Then $E$ is transversally parallelizable.

Proof. If we show that there exists an invariant metric on $E$, then the theorem will be a consequence of Lemmas 3.1 and 3.4. Let $Q$ be the orthogonal complement of $E$ in $T M$ relative to an arbitrary Riemannian $\tau$ on $T M$. Define $\sigma$ on $T M$ by

$$
\sigma=\tau \mid(E \times E) \oplus \sum_{i=1}^{m-r}\left(\beta_{i} \otimes \beta_{i}\right) .
$$

Clearly $\sigma$ is a Riemannian on $T M$. We show that $\sigma$ is invariant. First observe that for $\xi, \eta \in \Gamma(Q)$ and $X \in \Gamma(E)$,

$$
L_{X}\left(\sigma_{Q}(\xi, \eta)\right)=\sum_{i=1}^{m-r} L_{X}\left(\beta_{i}(\xi) \beta_{i}(\eta)\right)=\sum_{i=1}^{m-r}\left[\beta_{i}(\xi) L_{X}\left(\beta_{i}(\eta)\right)+\beta_{i}(\eta) L_{X}\left(\beta_{i}(\xi)\right)\right] .
$$

But

$$
\begin{aligned}
L_{X}\left(\beta_{i}(\eta)\right) & =L_{X}\left(i_{\eta} \beta_{i}\right)=i_{[X, \eta]} \beta_{i}+i_{y}\left(L_{X} \beta_{i}\right) \\
& =\beta_{i}([X, \eta])+i_{\eta}\left(\left[i_{X} d+d i_{X}\right]\left(\beta_{i}\right)\right)=\beta_{i}\left([X, \eta]_{Q}\right)=\beta_{i}\left(\tilde{V}_{X}(\eta)\right) .
\end{aligned}
$$

Thus

$$
\begin{aligned}
L_{X}\left(\sigma_{Q}(\xi, \eta)\right) & =\Sigma\left[\beta_{i}(\xi) \beta_{i}\left(\tilde{\nabla}_{X}(\eta)\right)+\beta_{i}(\eta) \beta_{i}\left(\tilde{\nabla}_{X}(\xi)\right)\right] \\
& =\sigma_{Q}\left(\xi, \tilde{\nabla}_{X} \eta\right)+\sigma_{Q}\left(\tilde{\nabla}_{X} \xi, \eta\right)
\end{aligned}
$$

as required. The theorem follows.

REMARK. In the proof of the preceding theorem we have introduced a new metric $\sigma=\left.\tau\right|_{E} \oplus \sum_{i=1}^{m-r}\left(\beta_{i} \otimes \beta_{i}\right)$. Observe that the orthogonal complement of $E$ relative to $\sigma$ is the same as for $\tau$, namely $Q$. The gradient vector fields of the 1 -forms $\beta_{1}, \beta_{2}, \cdots, \beta_{m-r}$ with respect to this metric are parallel along the leaves of $E$. In the following we will use these vector fields without specific refe- 
rences to the metric $\sigma$. Thus $\operatorname{grad} \beta_{i}$ is the unique section of $Q$ satisfying

$$
\sum_{j=1}^{m-r} \beta_{j}\left(\operatorname{grad} \beta_{i}\right) \beta_{j}(Y)=\beta_{i}(Y)
$$

for all $Y \in \Gamma(Q)$.

We make a few remarks regarding completeness. First note that if the metric $\tau$ is complete then the metric $\sigma$ will also be complete if there exist numbers $l$ and $L$ such that

$$
l \tau_{p}\left(X_{p}, X_{p}\right) \leqq \sum_{i=1}^{m-r} \beta_{i}\left(X_{p}\right)^{2} \leqq L \tau_{p}\left(X_{p}, X_{p}\right)
$$

for all $p \in M$ and $X \in \Gamma(Q)$. If this is the case then the vector fields $\left[1 / \beta_{i}\left(\operatorname{grad} \beta_{i}\right)\right] \operatorname{grad} \beta_{i}$ are complete vector fields. In any case (assuming $\tau$ is complete) the vector fields grad $\beta_{i}$ will be complete if they are bounded in the metric $\tau$. Moreover, in this case, every linear combination in the grad $\beta_{i}$ is complete.

COROLLARY 3.7. If in addition to the hypothesis of Theorem 3.5 we require that every linear combination of the vector fields grad $\beta_{i}$ (see 3.6) be complete, then

(1) any two leaves of $E$ are diffeomorphic and if any leaf of $E$ is closed in $M$ they all are,

(2) if $E$ admits a closed leaf then there is a fibre bundle $p$ : $M \rightarrow N$ where $N$ is parallelizable and $E$ is the foliation of $M$ whose leaves are the fibres of $p$.

Proof. The corollary follows immediately from Theorem 3.5 above and Propositions 4.3 and 4.4 of Conlon [5].

We now apply the results of this section to function groups.

As an example consider the case where $M$ is symplectic and suppose there is a Hamiltonian function $H \in C^{\infty}(M)$ such that $d H(p) \neq 0$ for each $p \in M$. Clearly $\{H\}$ globally generates a function group $\mathscr{H}$ of rank 1 . This leads to a foliation $E$ which is generated by the unique Hamiltonian vector field $X_{H}=X_{d H}$. The reciprocal function group $\mathscr{Z}^{\prime}$, which consists of all local integrals of $E$, also determines a foliation $E^{\prime}$. Thus by Theorem $3.5, E^{\prime}$ is transversally parallelizable. Indeed, if the vector field $\operatorname{grad}(d H)$ is complete then each two leaves of $E^{\prime}$ are diffeomorphic. Also since the leaves are the components of the level surfaces of $H$, they are closed and hence, by Corollary 3.7, they fibre $M$ over a parallelizable manifold. The following theorem generalizes this example where 
$M$ is not necessarily symplectic and the $\operatorname{grad} \beta_{i}$ are as defined by 3.6.

THEOREM 3.8. Let $\omega$ be a closed 2-form of constant rank $\rho$ on $M$. Let $\beta_{1}, \beta_{2}, \cdots, \beta_{r}$ be closed 1-forms which globally generate $a$ function group $\mathscr{S}$ of rank $r$. Then the foliation $E^{\prime}$ determined by the reciprocal function group of $\mathscr{S}$ is transversally parallelizable. Moreover, if every linear combination of the vector fields grad $\beta_{i}, i=1,2, \cdots, r$ is complete then $E^{\prime}$ is a complete transversally parallelizable foliation and each two leaves of $E^{\prime}$ are diffeomorphic. Furthermore, if one of the leaves of $E^{\prime}$ is closed then they all are and $M$ is a fibre bundle over a parallelizable manifold in which the fibres are the leaves of $E^{\prime}$.

Proof. The theorem is an immediate consequence of what it means for $\left\{\beta_{1}, \beta_{2}, \cdots, \beta_{r}\right\}$ to globally generate $\mathscr{S}$, Theorem 3.5 and Corollary 3.7.

REMARK. Suppose that in the above theorem we have $r=\rho$. In this case $E^{\prime}=\operatorname{ker} \omega$. Moreover, if some leaf $L$ of the foliation $E^{\prime}$ is closed then the manifold $M$ is fibered by $\pi: M \rightarrow N$ where $\pi^{-1}(x) \cong L$, for each $x$, and $N$, the manifold of leaves of ker $\omega$, is a symplectic manifold. This is true since $N_{p} \cong Q_{p}$ and $\omega \mid(Q \times Q)$ is nondegenerate.

REMARK. If in the above theorem $r=1$, then $E^{\prime}$ is a foliation of codimension 1 and thus by [5, Proposition 5.1] we conclude that either every leaf of $E^{\prime}$ is closed or else every leaf of $E^{\prime}$ is dense in $M$.

REMARK. If in addition to the hypothesis of the above theorem we assume that the 1 -forms $\beta_{1}, \beta_{2}, \cdots, \beta_{r}$ are exact, then there exist functions $H_{1}, H_{2}, \cdots, H_{r}$ such that $d H_{i}=\beta_{i}$ and the leaves of $E^{\prime}$, being components of level surfaces of $H_{i}=h_{i}$, are necessarily closed. Thus we see that if the functions $\left\{H_{1}, H_{2}, \cdots, H_{r}\right\}$ globally generate a function group of rank $r$ and every linear combination of the grad $\left(H_{i}\right)$ is complete then each two components of the level surfaces $H_{i}=h_{i}$ are diffeomorphic.

ACKNOWLEDGEMENT. The authors would like to express their gratitude to David J. Simms for his many valuable comments.

\section{REFERENCES}

1. R. Abraham and J. Marsden, Foundations of Mechanics, W. A. Benjamin, Inc., New York, 1967. 
2. M. Andrié and D. J. Simms, Constants of motion and Lie group actions, J. Math. Phys., 13 (1972) 331-336.

3. V. I. Arnold and A. Avez, Ergodic Problems of Classical Mechanics, W. A. Benjamin, Inc., New York, 1968.

4. C. Carathéodory, Calculus of Variations and Partial Differential Equations of the First Order Part I: Partial Differential Equations of the First Order, Holden-Day Series in Mathematical Physics, San Francisco, 1965.

5. L. Conlon, Transversally parallelizable foliations of condimension two, Trans. Amer. Math. Soc., 194 (1974), 79-102.

6. L. P. Eisenhart, Continuous Groups of Transformations, Dover Publications, Inc., New York, 1933.

7. W. Greub, S. Halperin and R. Vanstone, Connections, Curvature, and Cohomology, Volumes I and II, Academic Press Series in Pure and Applied Mathematics, New York, 1972-73.

8. R. Hermann, On the differential geometry of foliations, Ann. of Math. 72 (1960) $445-457$.

9. - Lie Algebras and Quantum Mechanics, W. A. Benjamin, Inc., New York, 1970.

10. O. Loos, Lie transformation groups of Banach manifolds, J. Differential Geome. 5 (1971), 175-185.

11. R. Palais, A global formulation of the Lie theory of transformation groups, Mem.

22 Amer. Math. Soc., Providence, R. I., 1957.

12. B. L. Reinhart, Foliated manifolds with bundle-like metrics, Ann. of Math. 69 (1959) 119-132.

Received December 11, 1975 and in revised form July 6, 1976.

North Carolina State University 



\section{PACIFIC JOURNAL OF MATHEMATICS}

\section{EDITORS}

RICHARD ARENS (Managing Editor)

University of California

Los Angeles, California 90024

\section{J. DugunduI}

Department of Mathematics University of Southern California Los Angeles, California 90007

D. Gilbarg and J. Milgram

Stanford University

Stanford, California 94305

\author{
R. A. Beaumont \\ University of Washington \\ Seattle, Washington 98105
}

\section{ASSOCIATE EDITORS}
B. H. NeUmanN
F. WOLF
K. YosHIDA

\section{SUPPORTING INSTITUTIONS}

\author{
UNIVERSITY OF BRITISH COLUMBIA \\ CALIFORNIA INSTITUTE OF TECHNOLOGY \\ UNIVERSITY OF CALIFORNIA \\ MONTANA STATE UNIVERSITY \\ UNIVERSITY OF NEVADA \\ NEW MEXICO STATE UNIVERSITY \\ OREGON STATE UNIVERSITY \\ UNIVERSITY OF OREGON \\ OSAKA UNIVERSITY
}

\author{
UNIVERSITY OF SOUTHERN CALIFORNIA \\ STANFORD UNIVERSITY \\ UNIVERSITY OF HAWAII \\ UNIVERSITY OF TOKYO \\ UNIVERSITY OF UTAH \\ WASHINGTON STATE UNIVERSITY \\ UNIVERSITY OF WASHINGTON \\ AMERICAN MATHEMATICAL SOCIETY
}

The Supporting Institutions listed above contribute to the cost of publication of this Journal, but they are not owners or publishers and have no responsibility for its content or policies.

Mathematical papers intended for publication in the Pacific Journal of Mathematics should be in typed form or offset-reproduced, (not dittoed), double spaced with large margins. Please do not use built up fractions in the text of your manuscript. You may however, use them in the displayed equations. Underline Greek letters in red, German in green, and script in blue. The first paragraph or two must be capable of being used separately as a synopsis of the entire paper. Items of the bibliography should not be cited there unless absolutely necessary, in which case they must be identified by author and Journal, rather than by item number. Manuscripts, in triplicate, may be sent to any one of the editors. Please classify according to the scheme of Math. Reviews, Index to Vol. 39. All other communications should be addressed to the managing editor, or Elaine Barth, University of California, Los Angeles, California, 90024.

The Pacific Journal of Mathematics expects the author's institution to pay page charges, and reserves the right to delay publication for nonpayment of charges in case of financial emergency.

100 reprints are provided free for each article, only if page charges have been substantially paid. Additional copies may be obtained at cost in multiples of 50 .

The Pacific Journal of Mathematics is issued monthly as of January 1966. Regular subscription rate: $\$ 72.00$ a year (6 Vols., 12 issues). Special rate: $\$ 36.00$ a year to individual members of supporting institutions.

Subscriptions, orders for back numbers, and changes of address should be sent to Pacific Journal of Mathematics, 103 Highland Boulevard, Berkeley, California, 94708.

PUBLISHED BY PACIFIC JOURNAL OF MATHEMATICS, A NON-PROFIT CORPORATION

Printed at Kokusai Bunken Insatsusha (International Academic Printing Co., Ltd.), 8-8, 3-chome, Takadanobaba, Shinjuku-ku, Tokyo 160, Japan. 


\section{Pacific Journal of Mathematics}

Patricia Andresen and Marvin David Marcus, Weyl's inequality and

quadratic forms on the Grassmannian .......................

George Bachman and Alan Sultan, Regular lattice measures: mappings and

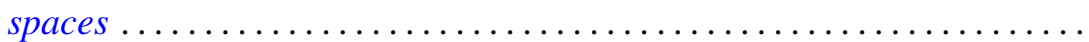

David Geoffrey Cantor, On certain algebraic integers and approximation by rational functions with integral coefficients ...................

James Richard Choike, On the value distribution of functions meromorphic in the unit disk with a spiral asymptotic value ..................

David Earl Dobbs, Divided rings and going-down................ 353

Mark Finkelstein and Robert James Whitley, Integrals of continuous

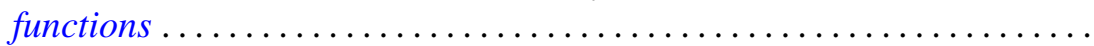

Ronald Owen Fulp and Joe Alton Marlin, Integrals of foliations on manifolds with a generalized symplectic structure ...............

Cheong Seng Hoo, Principal and induced fibrations .................

Wu-Chung Hsiang and Richard W. Sharpe, Parametrized surgery and

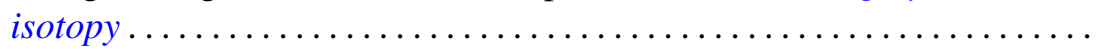

Surender Kumar Jain, Surjeet Singh and Robin Gregory Symonds, Rings whose proper cyclic modules are quasi-injective .................

Pushpa Juneja, On extreme points of the joint numerical range of commuting normal operators...

Athanassios G. Kartsatos, Nth order oscillations with middle terms of order $N-2$.

John Keith Luedeman, The generalized translational hull of a

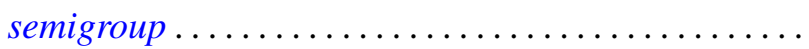

Louis Jackson Ratliff, Jr., The altitude formula and DVR's ...

Ralph Gordon Stanton, C. Sudler and Hugh C. Williams, An upper bound for the period of the simple continued fraction for $\sqrt{D}$...

David Westreich, Global analysis and periodic solutions of second order systems of nonlinear differential equations...

David Lee Armacost, Correction to: "Compactly cogenerated LCA

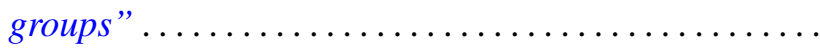

Jerry Malzan, Corrections to: "On groups with a single involution" .

David Westreich, Correction to: "Bifurcation of operator equations with unbounded linearized part" ...................... 